INVESTIGACIÓN

\title{
INFLUENCIA DEL TIPO DE CONTRATACIÓN EN EL CLIMA ORGANIZACIONAL: UNA EVIDENCIA EMPÍRICA EN LA UNIVERSIDAD
}

\section{INFLUENCE OF THE TYPE OF CONTRACTING IN THE ORGANIZATIONAL CLIMATE: AN EMPIRICAL EVIDENCE IN THE UNIVERSITY}

Administradora de Empresas. Doralba Coronel Coronel ${ }^{\mathrm{a}}$, Administradora de Empresas. Yenifer Tatiana Santiago Serna ${ }^{\mathrm{b}}$, Msc. José Gregorio Arévalo Ascanio ${ }^{\mathrm{c}}$

${ }^{a}$ Universidad Francisco de Paula Santander Ocaña, Grupo de Investigación GIDSE, Vía Acolsure Sede el Algodonal, Ocaña - Norte de Santander, Colombia, dcoronelc@ufpso.edu.co.edu.co

${ }^{\mathrm{b}}$ Universidad Francisco de Paula Santander Ocaña, Grupo de Investigación GIDSE, Vía Acolsure Sede el Algodonal, Ocaña - Norte de Santander, Colombia, ytsantiagos@ufpso.edu.co

${ }^{c}$ Universidad Francisco de Paula Santander Ocaña, Grupo de Investigación GIDSE, Vía Acolsure Sede el Algodonal, Ocaña - Norte de Santander, Colombia, jgarevaloa@ufpso.edu.co

Fecha de recepción: 15-03-2017

Fecha de aprobación: 01-06-2017

Resumen: Este artículo tiene como objetivo presentar algunos de los resultados obtenidos en el proyecto titulado "Influencia del tipo de contratación en el clima organizacional del área administrativa de la Universidad Francisco de Paula Santander Ocaña", direccionados fundamentalmente hacia la medición del clima laboral en la institución con relación a las dimensiones: autonomía, cohesión, confianza, presión, apoyo, reconocimiento, equidad e innovación. La metodología utilizada se basó en un estudio cuantitativo por medio de la aplicación de encuestas individuales que permitieron el análisis y la presentación de los resultados.

Palabras clave: Clima organizacional, clima laboral, entorno de trabajo, universidad. 


\begin{abstract}
This article aims to present some of the results obtained in the project entitled "Influence of the type of hiring in the organizational climate of the administrative area of the University Francisco de Paula Santander Ocaña", directed mainly towards the measurement of the working environment in the institution in relation to the dimensions: autonomy, Cohesion, trust, pressure, support, recognition, equity and innovation. The methodology used was based on a quantitative study through the application of individual surveys that allowed the analysis and presentation of the results.
\end{abstract}

Keywords: Organizational climate, work climate, work environment, university.

\section{INTRODUCCIÓN}

El clima organizacional viene siendo un evento de estudio en el que se ha venido investigando desde varios años atrás por parte de la comunidad científica, situación que evidencia que este tema ha pasado a convertirse en un componente clave de la dinámica organizacional. De acuerdo con Fuente especificada no válida. el clima organizacional es hoy un factor clave en el desarrollo empresarial, y su estudio en profundidad, diagnóstico y mejoramiento incide de manera directa en el denominado espíritu de la organización.

Ahora bien, en el marco del proyecto de investigación "Influencia del tipo de contratación en el clima organizacional del área administrativa de la Universidad Francisco de Paula Santander Ocaña", en el que uno de sus objetivos fue realizar una medición del clima laboral en la institución con base en las dimensiones de autonomía, cohesión, confianza, presión, apoyo, reconocimiento, equidad e innovación con el fin de identificar la influencia del tipo de contratación, para este propósito se aplicó un cuestionario a los empleados administrativos y de planta de la institución de educación superior objeto de estudio.

El presente artículo se estructura en las siguientes partes: una primera en la que se presenta una revisión de algunas investigaciones sobre clima organizacional en el contexto educativo. En la segunda parte se muestra que la metodología empleada fue de carácter cuantitativo a través de una investigación de campo en la que se aplicó un instrumento de recolección de datos tipo encuesta a las diferentes categorías de empleados de la institución.

La tercera parte hace alusión al análisis de los resultados de la información recolectada, en los que se constata que de las ocho (8) dimensiones consideradas para evaluar el clima laboral de la universidad solo tres (confianza, presión y reconocimiento) se vieron afectadas por el tipo de contratación; y por último se exponen las principales conclusiones de la investigación.

\section{INVESTIGACIONES SOBRE CLIMA ORGANIZACIONAL EN EL CONTEXTO EDUCATIVO.}

Con el propósito de explorar los estudios sobre clima organizacional, se realizó una revisión sistemática en Redalyc, Scielo y Science Direct, a partir de categorías como: clima organizacional, clima laboral y entorno de trabajo y se establecieron los siguientes límites: artículos publicados en revistas, así como tesis de universidades cuya temática fuera aplicable al contexto educativo. 
Entre los trabajos que están directamente relacionados con la investigación realizada, se destaca el de (Naranjo , Paz, \& Marín, 2014), cuyo objetivo consistió en determinar los procesos de intervención del clima organizacional en la Institución prestadora de servicios de salud (IPS) de la Universidad Autónoma de Manizales, para dicho propósito realizaron un estudio descriptivo, transversal y observacional, con un universo de 84 trabajadores activos en la IPS., encontrándose como resultado más importante que existe una percepción poco satisfactoria del clima organizacional, especialmente en el servicio de odontología de la (IPS).

Por otro lado, en un estudio efectuado por (Tapias, 2014), en el área administrativa de la Universidad Tecnológica del Chocó, en la que se aplicaron 120 cuestionarios a empleados de la organización objeto de estudio; se arrojaron puntuaciones como: autonomía $72 \%$, cohesión $57 \%$, apoyo $51 \%$, confianza 47\%, innovación 42\%, presión $34 \%$ reconocimiento $33 \%$ y equidad $27 \%$. De acuerdo con este resultado se pudo afirmar que el clima organizacional en la U.T.CH, es mejorable.

Por su parte, la investigación de (Ucros, 2011) expone los resultados del análisis de los factores del clima organizacional en las universidades de la Costa Caribe Colombiana, en las se pudo evidenciar una aproximación comparativa de los factores psicológicos individuales, grupales y organizacionales, los cuales indican que hay cuestionamientos que afectan el clima organizacional en las universidades analizadas.

Asimismo, en el trabajo de (Brito \& Jiménez, 2009), llevado a cabo en una universidad venezolana, en la que se aplicaron instrumentos a personal docente, administrativo y obrero de la institución; se encontró que la situación actual del clima organizacional de la unidad de análisis se ve afectada por algunas deficiencias en la gestión institucional, y en lo que se refiere cooperación laboral, por parte del personal, sin embargo, la gran mayoría de los individuos encuestados mostraron identificación y orgullo de pertenecer a la universidad.

Del mismo modo, se halló el trabajo de (Bermúdez, Pedraza, \& Rincón, 2015), en el que los resultados evidenciaron, desde la percepción de los estudiantes, que el clima organizacional universitario es el conjunto de factores tangibles (infraestructura, recursos, etc.) e intangibles (valores, relaciones interpersonales, situaciones, etc.), que se viven a diario en la universidad.

Finalmente, se encontró el trabajo de (Juliao, Sánchez, \& Martínez, 2014), que a pesar de que no fue realizado en el contexto educativo, sí tiene que ver mucho con el presente manuscrito ya que su propósito fue demostrar que el tipo de contratación de una empresa de bebidas de la costa norte de Colombia que cuenta con trabajadores distribuidos en las ciudades de Barranquilla, Cartagena, Santa Marta, Sincelejo y Valledupar influye en el clima laboral.

\section{METODOLOGÍA.}

Se realizó una investigación descriptiva, la cual según (Hurtado, 2000) tiene como objetivo central lograr la descripción o caracterización del evento de estudio dentro de un contexto particular, es así que el presente estudio se centró en el enfoque cuantitativo, en tanto que usó la recolección de datos, con base en la medición numérica y el análisis estadístico, para establecer patrones de comportamiento y probar teorías (Hernández, Fernández, \& Baptista, 2006).

La población seleccionada para la realización de esta investigación son los empleados administrativos y de planta de la 
Universidad Francisco de Paula Santander Ocaña. La cual estuvo representada por una población finita como se nota en la tabla 1.

\begin{tabular}{|c|c|}
\hline Administrativos & 28 \\
\hline Contrato a término fijo & 221 \\
\hline $\begin{array}{c}\text { Orden de prestación de } \\
\text { servicios }\end{array}$ & 53 \\
\hline $\begin{array}{c}\text { Orden de prestación de } \\
\text { servicios profesionales }\end{array}$ & 66 \\
\hline Total & $\mathbf{3 6 8}$ \\
\hline
\end{tabular}

Fuente: Elaboración propia

Para esta investigación se llevó a cabo un tipo de muestreo estratificado, donde la población se dividió en 4 subgrupos de acuerdo a los tipos de contrato que implementa la institución.

n: Muestra

$$
\mathrm{n}=\frac{\mathrm{Z}^{2} \cdot \mathrm{N} \cdot \mathrm{P} \cdot \mathrm{Q}}{\mathrm{e}^{2}(\mathrm{~N}-1)+\mathrm{Z}^{2} \cdot P \cdot \mathrm{Q}}
$$

Z: Nivel de confianza

e: Margen de error

P: Proporción de aceptación

Q: Proporción de rechazo

$$
\begin{gathered}
\mathrm{n}=(1.96)^{2}(368)(0.50)(0.50) \\
(0.05)^{2}(368-1)+(1.96)^{2}(0.50)(0.50) \\
\mathrm{n}=188
\end{gathered}
$$

Para este caso se tomó una muestra de 188 personas la cual esta estratificada de la siguiente manera:

Administrativos: 14

Contratados a Término fijo: 113

Orden de Prestación de Servicios: 27

Orden de Prestación de Servicios Profesionales: 34

\section{RESULTADOS}

A continuación se presentan los resultados de la aplicación de la encuesta estructurada por ocho (8) dimensiones (Autonomía, cohesión, confianza, presión, apoyo, reconocimiento, equidad e innovación).

En la tabla 2 se aprecia los resultados obtenidos con respecto a la dimensión de autonomía.

Tabla 2.

Percepción de la dimensión autonomía

\begin{tabular}{lcccccccc}
\hline \multicolumn{1}{c}{ Autonomia } & \multicolumn{2}{c}{ OPS } & \multicolumn{2}{c}{ OPS } & Indefinido & \multicolumn{2}{c}{ Término Fijo } \\
& \multicolumn{2}{c}{ Profesionales } & & & & & & \\
\cline { 2 - 9 } & Total & $\%$ & Total & $\%$ & Total & $\%$ & Total & $\%$ \\
\hline Muyde acuerdo & 16 & 47.1 & 13 & 48.1 & 9 & 643 & 46 & 40.7 \\
De acuerdo & 10 & 29.4 & 12 & 44.4 & 4 & 28.6 & 54 & 47.8 \\
\hline No estoyseguro & 5 & 14.7 & 2 & 7.5 & 1 & 7.1 & 9 & 8.0 \\
\hline En desacuerdo & 2 & 5.9 & 0 & 0.0 & 0 & 0.0 & 3 & 2.6 \\
\hline Totalmente en & 0 & 0.0 & 0 & 0.0 & 0 & 0.0 & 0 & 0.0 \\
desacuerdo. & & & & & & & & \\
\hline No responde & 1 & 2.9 & 0 & 0.0 & 0 & 0.0 & 1 & 0.9 \\
\hline Total & 34 & 100 & 27 & 100 & 14 & 100 & 113 & 100 \\
\hline
\end{tabular}

La tabla 2 muestra la dimensión de autonomía de acuerdo al tipo de contratación

De acuerdo con (Chiang, Salazar, Huerta, \& Nuñez , 2008) la autonomía se puede definir como la percepción del trabajador acerca de la autodeterminación y responsabilidad necesaria en la toma de decisiones con respecto a procedimientos del trabajo, metas y prioridades. En la Universidad Francisco de Paula Santander Ocaña, los resultados señalan que en general se presenta una actitud favorable por parte de los empleados en los diferentes tipos de contrato frente a esta dimensión. Sin embargo, es importante resaltar que los funcionarios con contrato OPSP a pesar de organizar y evaluar su trabajo y de tener iniciativa laboral, sienten menos autonomía laboral ya que poseen poca libertad en la toma de decisiones. Por ultimo podemos concluir que en la mayoría de los aspectos tenidos en cuenta los empleados de contrato a término indefinido sienten mayor autonomía. 
Tabla 3.

Percepción de la dimensión cohesión

\begin{tabular}{lcccccccc}
\hline \multicolumn{1}{c}{ Cohesión } & \multicolumn{2}{c}{ OPS } & \multicolumn{2}{c}{ OPS } & Indefinido & \multicolumn{2}{c}{ Término Fijo } \\
& \multicolumn{2}{c}{ Profesionales } & & & & & & \\
\cline { 2 - 9 } & Total & $\%$ & Total & $\%$ & Total & $\%$ & Total & $\%$ \\
\hline Muy de acuerdo & 14 & 41.2 & 15 & 55.6 & 6 & 42.9 & 52 & 46.0 \\
\hline De acuerdo & 13 & 38.2 & 9 & 33.3 & 5 & 35.7 & 47 & 41.6 \\
\hline No estoy seguro & 4 & 11.9 & 2 & 7.4 & 2 & 14.3 & 12 & 10.6 \\
\hline En desacuerdo & 1 & 2.9 & 1 & 3.7 & 1 & 7.1 & 2 & 1.8 \\
\hline Totalmente en & 1 & 2.9 & 0 & 0.0 & 0 & 0.0 & 0 & 0.0 \\
desacuerdo. & & & & & & & & \\
\hline No responde & 1 & 2.9 & 0 & 0.0 & 0 & 0.0 & 0 & 0.0 \\
\hline Total & 34 & 100 & 27 & 100 & 14 & 100 & 113 & 100 \\
\hline
\end{tabular}

La tabla 3 muestra la dimensión de cohesión de acuerdo al tipo de contratación

Para Litwin y Stinger citado en (Vargas, 2010) la cohesión es la percepción de los miembros de la empresa acerca de la existencia de un ambiente de trabajo grato y de buenas relaciones sociales tanto entre pares como entre jefes y subordinados. En la Universidad la cohesión se encuentra en un nivel positivo, ya que, tanto los empleados de contrato a término indefinido como los de contrato por OPS, término fijo y OPS profesional manifiestan estar cómodos con las relaciones establecidas. Sin embargo, se puede apreciar que los trabajadores de contrato a término fijo y orden por prestación de servicios (OPS) frente a los empleados de contrato a término indefinido y OPSP son quienes tienen mayor grado de compañerismo, promueven un ambiente amigable dentro de la institución y existe un espíritu de trabajo en equipo, lo que permite que exista ayuda y colaboración entre todos.

Según (Ministerio de Salud, 2009)la confianza se basa en las redes de comunicación que existen dentro de la organización así como la facilidad que tienen los empleados de hacer que se escuchen sus opiniones en la dirección. Esta dimensión en el plantel educativo es satisfactoria, ya que todos los funcionaros de las diferentes modalidades de contratación afirma estar de acuerdo en que existe buena comunicación y relación entre los superiores y subordinados; la dirección tiene confianza condescendiente para con los empleados y existe confianza entre los compañeros de trabajo. No obstante, los trabajadores con contrato por orden de prestación de servicios profesionales (OPSP) a pesar de estar en acuerdo con los ítems de esta dimensión presentan un porcentaje menor en relación a los contratos de término indefinido y término fijo.

Tabla 4.

Percepción de la dimensión confianza

\begin{tabular}{lcccccccc}
\hline \multicolumn{1}{c}{ Confianza } & \multicolumn{2}{c}{ OPS } & \multicolumn{2}{c}{ OPS } & & Indefinido & \multicolumn{2}{c}{ Término Fijo } \\
& \multicolumn{2}{c}{ Profesionales } & & & & & & \\
\cline { 2 - 9 } & Total & $\%$ & Total & $\%$ & Total & $\%$ & Total & $\%$ \\
\hline Muy de acuerdo & 11 & 32.4 & 13 & 48.1 & 8 & 57.0 & 42 & 37.2 \\
De acuerdo & 16 & 47.1 & 11 & 40.7 & 5 & 36.0 & 57 & 50.4 \\
\hline No estoy seguro & 6 & 17.6 & 3 & 11.1 & 0 & 0.0 & 11 & 9.7 \\
\hline En desacuerdo & 1 & 2.9 & 0 & 0 & 1 & 7.0 & 2 & 1.8 \\
\hline Totalmente en & 0 & 0.0 & 0 & 0 & 0 & 0.0 & 0 & 0.0 \\
desacuerdo. & & & & & & & & \\
\hline No responde & 0 & 0.0 & 0 & 0 & 0 & 0.0 & 1 & 0.9 \\
\hline Total & 34 & 100 & 27 & 100 & 14 & 100 & 113 & 100 \\
\hline
\end{tabular}

La tabla 4 muestra la dimensión de confianza de acuerdo al tipo de contratación.

Tabla 5.

Percepción de la dimensión presión

\begin{tabular}{lcccccccc}
\hline \multirow{3}{*}{ Presión } & \multicolumn{3}{c}{ OPS } & \multicolumn{2}{c}{ OPS } & Indefinido & \multicolumn{2}{c}{ Término Fijo } \\
& \multicolumn{1}{c}{ Profesionales } & & & & & & \\
\cline { 2 - 10 } & Total & $\%$ & Total & $\%$ & Total & $\%$ & Total & $\%$ \\
\hline Muy de acuerdo & 4 & 11.8 & 3 & 11.1 & 5 & 35.7 & 17 & 15.0 \\
\hline De acuerdo & 7 & 20.6 & 8 & 29.6 & 2 & 14.3 & 37 & 32.7 \\
\hline No estoy seguro & 8 & 23.5 & 7 & 25.9 & 3 & 21.5 & 21 & 18.7 \\
\hline En desacuerdo & 12 & 35.3 & 5 & 18.5 & 2 & 14.3 & 24 & 21.2 \\
\hline Totalmente en & 2 & 5.9 & 4 & 14.9 & 1 & 7.1 & 14 & 12.4 \\
desacuerdo. & & & & & & & & \\
\hline No responde & 1 & 2.9 & 0 & 0.0 & 1 & 7.1 & 0 & 0.0 \\
\hline Total & 34 & 100 & 27 & 100 & 14 & 100 & 113 & 100 \\
\hline
\end{tabular}

La tabla 5 muestra la dimensión de presión de acuerdo al tipo de contratación. 
Para Koys y DeCotiis, citados en (Frìas, Carranza, \& Perez, 2015) la presión se refiere a la exigencia de tiempo respecto a la ejecución de una tarea y a unos estándares de rendimiento que la organización impone a sus colaboradores. En la institución de educación superior objeto de estudio, la dimensión de presión tiene mayor influencia en el contrato a término indefinido y término fijo, pues los trabajadores sienten que tienen mucho trabajo y poco tiempo para realizarlo, lo que genera que se sientan estresados y ansiosos con las responsabilidades que tiene a su cargo y por ende sienten como si nunca tuviesen un día libre. Mientras que los demás contratos (OPS y OPSP) coinciden en que el tiempo para realizar sus labores es suficiente y el estrés que manejan de acuerdo con sus responsabilidades de trabajo es muy bajo, pero hay empleados en todos las modalidades de contratación que no están seguros de la influencia que tiene esta dimensión en su puesto de trabajo; además la mayoría de trabajadores aseguran que la universidad es un lugar relajado para trabajar.

Tabla 6.

$\begin{array}{cl}\text { Percepción de la dimensión apoyo } & \\ \text { OPS OPS Indefinido Témino Fijo }\end{array}$

\begin{tabular}{lcccccccc}
\multirow{2}{*}{ Apoyo } & \multicolumn{2}{c}{ Profesionales } & & & & & \\
\cline { 2 - 9 } & Total & $\%$ & Total & $\%$ & Total & $\%$ & Total & $\%$ \\
\hline Muy de acuerdo & 10 & 29.4 & 11 & 40.7 & 8 & 57.1 & 46 & 40.7 \\
\hline De acuerdo & 13 & 38.2 & 11 & 40.7 & 5 & 35.8 & 51 & 45.1 \\
\hline No estoy seguro & 6 & 17.6 & 3 & 11.2 & 1 & 7.1 & 13 & 11.5 \\
\hline En desacuerdo & 1 & 2.9 & 1 & 3.7 & 0 & 0.0 & 2 & 1.8 \\
\hline Totalmente en & 4 & 11.9 & 1 & 3.7 & 0 & 0.0 & 1 & 0.9 \\
desacuerdo. & & & & & & & & \\
\hline No responde & 0 & 0.0 & 0 & 0.0 & 0 & 0.0 & 0 & 0.0 \\
\hline Total & 34 & 100 & 27 & 100 & 14 & 100 & 113 & 100 \\
\hline
\end{tabular}

La tabla 6 muestra la dimensión de apoyo de acuerdo al tipo de contratación

Para Mercado y Toro citado en (Palacios \& Vargas, 2015) el apoyo es la percepción del grado en que los compañeros se respaldan y el jefe estimula y da participación a sus colaboradores. En la universidad se observa un resultado positivo en esta dimensión en todos los tipos de contratos (indefinido, fijo, OPS y OPSP), puesto que se logra un respaldo entre los jefes y los empleados motivándoles a superarse cada día más y cada jefe cuida y trata con respeto al personal que tiene a su cargo. Aunque, los funcionarios contratados por OPSP a pesar de presentar una actitud favorable frente a esta variable los porcentajes de los resultados son menores con respecto a las demás modalidades de contratación.

Tabla 7.

Percepción de la dimensión reconocimiento La tabla 7 muestra la dimensión de reconocimiento

\begin{tabular}{lcccccccc}
\hline \multirow{2}{*}{ Reconocimiento } & \multicolumn{2}{c}{ OPS } & \multicolumn{2}{c}{ OPS } & \multicolumn{1}{c}{ Indefinido } & \multicolumn{2}{c}{ Témino Fijo } \\
& \multicolumn{1}{c}{ Profesionales } & & & & & & \\
\cline { 2 - 10 } & Total & $\%$ & Total & $\%$ & Total & $\%$ & Total & $\%$ \\
\hline Muy de acuerdo & 9 & 26.5 & 8 & 29.6 & 6 & 42.9 & 24 & 21.2 \\
\hline De acuerdo & 9 & 26.5 & 9 & 33.3 & 6 & 42.9 & 55 & 48.7 \\
\hline No estoy seguro & 10 & 29.4 & 6 & 22.3 & 1 & 7.1 & 25 & 22.1 \\
\hline En desacuerdo & 4 & 11.8 & 2 & 7.4 & 1 & 7.1 & 6 & 5.3 \\
\hline Totalmente en & 2 & 5.8 & 2 & 7.4 & 0 & 0.0 & 2 & 1.8 \\
desacuerdo. & & & & & & & & \\
\hline No responde & 0 & 0.0 & 0 & 0.0 & 0 & 0.0 & 1 & 0.9 \\
\hline Total & 34 & 100 & 27 & 100 & 14 & 100 & 113 & 100 \\
\hline \multicolumn{7}{c}{ de acuerdo al tipo de contratación. } \\
\end{tabular}

Gandy citado en (Gonzales, 2015)señala que el reconocimiento laboral se da cuando se reconoce y valora a los empleados por su trabajo, por lo que ellos son y por el papel que desempeñan en sus organizaciones. En la universidad la dimensión de reconocimiento es favorable por que la gran mayoría de los empleados de los diferentes tipos de contratos manifiestan estar de acuerdo con que se estimula la capacitación de los funcionarios, se les reconoce cuando se hace un buen trabajo y se ofrecen posibilidades para su desarrollo personal. Sin embargo, se puede observar que hay un 
porcentaje significativo de empleados que no están seguros de recibir algún reconocimiento por su desempeño laboral. Así mismo, los empleados por OPSP, OPS y término fijo a pesar de que respondieron favorablemente a esta dimensión son los que presentan menor grado de reconocimiento frente a los trabajadores de contrato a término indefinido.

Tabla 8.

Percepción de la dimensión equidad

\begin{tabular}{lcccccccc}
\hline \multirow{2}{*}{ Equidad } & \multicolumn{2}{c}{ OPS Profesionales } & \multicolumn{2}{c}{ OPS } & \multicolumn{3}{c}{ Indefinido } & \multicolumn{3}{c}{ Término Fijo } \\
\cline { 2 - 10 } & Total & $\%$ & Total & $\%$ & Total & $\%$ & Total & $\%$ \\
\hline Muy de acuerdo & 9 & 26.5 & 10 & 37.0 & 7 & 50.0 & 39 & 34.5 \\
\hline De acuerdo & 17 & 50.0 & 10 & 37.0 & 4 & 28.6 & 51 & 45.1 \\
\hline No estoy seguro & 5 & 14.7 & 5 & 18.6 & 1 & 7.1 & 15 & 13.3 \\
\hline En desacuerdo & 1 & 2.9 & 1 & 3.7 & 2 & 14.3 & 4 & 3.5 \\
\hline $\begin{array}{l}\text { Totalmente en } \\
\text { desacuerdo. }\end{array}$ & 2 & 5.9 & 1 & 3.7 & 0 & 0.0 & 2 & 1.8 \\
\hline No responde & 0 & 0.0 & 0 & 0.0 & 0 & 0.0 & 2 & 1.8 \\
\hline Total & 34 & 100 & 27 & 100 & 14 & 100 & 113 & 100 \\
\hline
\end{tabular}

La tabla 8 muestra la dimensión de equidad de acuerdo al tipo de contratación.

Esta dimensión es entendida por (Hesse, Gómez, \& Bonales, 2010) , como la idea que reconoce que los seres humanos tienen el mismo valor aunque existan diferencias biológicas entre ellos. En la caso de la institución objeto de análisis se encontró que la dimensión de equidad es favorable en cada una de las modalidades de contratación (OPSP, OPS, indefinido y fijo), ya que los trabajadores afirman que reciben un trato justo por parte de su jefe, los objetivos fijados por su superior son razonables y a todos los empleados de la institución se les trata por igual.
Tabla 9. Percepción de la dimensión innovación

\begin{tabular}{|c|c|c|c|c|c|c|c|c|}
\hline \multirow{3}{*}{ Innovación } & \multicolumn{2}{|c|}{ OPS } & \multicolumn{2}{|c|}{ OPS } & \multicolumn{2}{|c|}{ Indefinido } & \multicolumn{2}{|c|}{ Término } \\
\hline & \multicolumn{2}{|c|}{ Profesionales } & & & & & \multicolumn{2}{|c|}{ Fijo } \\
\hline & Total & $\%$ & Total & $\%$ & Total & $\%$ & Total & $\%$ \\
\hline Muy de acuerdo & 11 & 32.4 & 11 & 40.7 & 6 & 42.9 & 38 & 33.6 \\
\hline De acuerdo & 15 & 44.1 & 11 & 40.7 & 6 & 42.9 & 63 & 55.8 \\
\hline No estoy & 5 & 14.7 & 3 & 11.2 & 1 & 7.1 & 10 & 8.8 \\
\hline En desacuerdo & 2 & 5.9 & 1 & 3.7 & 1 & 7.1 & 2 & 1.8 \\
\hline $\begin{array}{l}\text { Totalmente en } \\
\text { desacuerdo. }\end{array}$ & 1 & 2.9 & 1 & 3.7 & 0 & 0.0 & 0 & 0.0 \\
\hline No responde & 0 & 0.0 & 0 & 0.0 & 0 & 0.0 & 0 & 0.0 \\
\hline Total & 34 & 100 & 27 & 100 & 14 & 100 & 113 & 100 \\
\hline
\end{tabular}

La tabla 9 muestra la dimensión de innovación de acuerdo al tipo de contratación

Según (Ministerio de Salud, 2009) la dimensión de innovación cubre la voluntad de una organización de experimentar cosas nuevas y de cambiar la forma de hacerlas. Para el caso de la universidad se percibe una gran disponibilidad por parte de los empleados de los diferentes tipos de contrato a la hora de afrontar cambios que sean necesarios para el crecimiento de la misma. Esta variable se encuentra en un nivel positivo, ya que, la mayoría de los empleados de cada una de las modalidades de contratación coinciden en estar de acuerdo con que los superiores valoran nuevas formas para hacer las cosas y les animan a encontrar nuevas formas de solucionar problemas. Además la institución de educación superior continuamente adopta nuevos y mejores métodos para realizar el trabajo.

\section{CONCLUSIONES.}

Los resultados obtenidos permiten plantear las siguientes conclusiones:

Es indudable que el clima organizacional ha pasado a convertirse en un componente clave de estudio de cualquier organización 
empresarial, por lo tanto es también importante que en las instituciones de educación superior se evalúe permanentemente el clima laboral para que esto ayude a mejorar el factor de bienestar institucional que se requiere para las pretensiones de acreditación de una universidad.

De las ocho (8) dimensiones consideradas para evaluar el clima laboral de la universidad solo tres (confianza, presión y reconocimiento) se vieron afectadas por el tipo de contratación; ya que, existe poca libertad para comunicarse abiertamente con los superiores, los empleados tienen mucho trabajo y poco tiempo para realizarlo generando estrés y ansiedad en los mismos y hay funcionarios que no reciben atención ni reconocimiento. Sin embargo estás no presentaron gran variación con respecto a las otras cinco (5) dimensiones. Por este motivo se puede concluir que las diferentes modalidades de contratos no afectan directamente el ambiente laboral en la universidad objeto de estudio.

Los datos obtenidos en la encuesta permiten afirmar que el clima laboral de la universidad presenta una actitud favorable, por este motivo se puede concluir que las condiciones laborales son aptas para que los empleados realicen sus funciones. Por medio de la encuesta se pudo identificar las variables que no permiten que el clima laboral sea totalmente favorable, esto fue de gran utilidad para realización de un plan de mejoramiento que permita trabajar directamente las variables que en este momento se encuentran afectadas.

En general, el clima laboral que se percibe al interior de la universidad, es de un ambiente moderadamente bueno, no obstante, este es muy cambiante debido a los múltiples factores externos que se viven actualmente.

\section{REFERENCIAS}

Bermúdez, J., Pedraza, A., \& Rincón, C. (2015). El clima organizacional en universidades de Bogotá desde la perspectiva de los estudiantes. Revista Electrónica de Investigación Educativa, 1-12.

Brito, Y., \& Jiménez, J. (2009). Evaluación del Clima Organizacional Universitario. Caso: Facultad de Ingeniería - Universidad de Carabobo. Ingeniería Industrial. Actualidad y Nuevas Tendencias, 7280 .

Chiang , M., Salazar, C., Huerta , P., \& Nuñez , A. (2008). Clima organizacional y satisfacción laboral en organizaciones del sector estatal (Instituciones públicas) Desarrollo, adaptación y validación de instrumentos. Universum.

Frìas, M., Carranza, G., \& Perez, A. (9 de Octubre de 2015). Universidad Autonoma de Guadalajara. Recuperado el 25 de Mayo de 2016, de

http://congreso.investiga.fca.unam.m $\mathrm{x} / \mathrm{docs} / \mathrm{xx} / \mathrm{docs} / 3.12 . \mathrm{pdf}$

García, M. (2009). Clima Organizacional y su Diagnóstico: Una aproximación Conceptual. Cuadernos de Administración(42), 43-61.

Gonzales, W. (2015). Biblioteca um. Recuperado el 20 de Mayo de 2016, de

http://dspace.biblioteca.um.edu.mx/js pui/bitstream/123456789/443/1/Tesis $\% 20 \mathrm{Mtr} \% \mathrm{C} 3 \%$ ADa.\%20Willie\%20 Gonzales.pdf 
Hernández, R., Fernández, C., \& Baptista, P. (2006). Metodología de la investigación. México: Mc Graw Hill.

Hesse, H., Gómez, R., \& Bonales, J. (2010). Clima organizacional de una institución pública de educación superior en Morelia, Michoacán, México. Escenarios, 41-50.

Hurtado, J. (2000). Metodología de la investigación holística. Caracas: Sypal.

Juliao, D., Sánchez, C., \& Martínez, D. (2014). Diferencias del clima laboral entre empleados contratados por outsourcing y empleados directos: evidencia empírica de la zona norte de Colombia. Universidad \& Empresa, 281-299.

Ministerio de Salud. (2009). Metodología para el Estudio del Clima Organizacional. Lima: J.W.G. Servicios Gráficos.

Ministerio de Salud. (2009). minsa.gob.pe. Recuperado el 20 de Mayo de 2016, de http://www.minsa.gob.pe/DGSP/cli ma/archivos/metodologia_clima.pdf

Naranjo , C., Paz, A., \& Marín, S. (2014). Clima organizacional: una investigacion en la Institución prestadores de Servicio de Salud (IPS) de la Universidad Autónoma de Manizales. Universidad \& Empresa, 105-126.

Palacios, F., \& Vargas, F. (2015). Unad.edu.co. Recuperado el 15 de Mayo de 2016, de http://repository.unad.edu.co/bitstrea m/10596/6148/1/9097421.pdf
Tapias, A. (2014). Diagnóstico del clima organizacional en área administrativa de la Universidad Tecnológica del Chocó. Bogotá: Universidad Nacional de Colombia.

Ucros, M. (2011). Factores del clima organizacional en las universidades de la Costa Caribe Colombiana. Omnia, 91-102.

Vargas, J. (2010). repositorio.ufp.edu.co. Recuperado el 20 de Mayo de 2016, de http://repositorio.utp.edu.co/dspace/b itstream/11059/1892/1/65838V297.p df 